\title{
Frequency-Swapping Aided Femtocells in Twin-Layer Cellular Networks Relying on Fractional Frequency Reuse
}

\author{
Fan Jin, Rong Zhang and Lajos Hanzo \\ School of ECS, University of Southampton, SO17 1BJ, United Kingdom. \\ Tel: +44-23-8059 3125, Fax: +44-23-8059 4508 \\ Email: \{fj1g10,rz,lh\}@ecs.soton.ac.uk, http://www-mobile.ecs.soton.ac.uk
}

\begin{abstract}
Femtocells constitute an economical solution conceived for improving the indoor coverage, which are capable of achieving a high network capacity. In order to guarantee a high Area Spectral Efficiency (ASE), femtocells have to reuse the spectrum of macrocells. As a result, the performance of both the femtocells and macrocells may suffer owing to the near-far effects. In this paper, we investigate the Outage Probability (OP) of twin-layer cellular networks, where the Macrocell Base Stations (MBSs) employing Fractional Frequency Reuse (FFR) host the Femtocell Base Stations (FBSs). More explicitly, the frequencyswapping aided femtocell concept is proposed for overcoming the typical near-far problem. We derive the approximate closed-form expressions for the DownLink (DL) OP for both our benchmarker as well as for our proposed solution. Our analysis demonstrates that the OP of femtocell users in the Cell Centre Region (CCR) and that of the macrocell users in the Cell Edge Region (CER) will be reduced by the proposed swapped-spectrum access policy.
\end{abstract}

\section{INTRODUCTION}

Femtocells may be viewed as low-power access points, which have the potential of providing high-quality network access for indoor users at a low cost. Hence they constitute a cost-effective way of reducing the traffic of the entire cellular system [1]. Femtocells may be overlaid onto macrocells, hence forming a hierarchical twin-layer network structure [2]. Unlike the macrocells, which are designed for predetermined geographic locations and theoretically modelled as a regular tessellated hexagonal lattice, femtocells are constructed rather randomly. When the femtocells reuse the frequencies occupied by the over-sailing macrocells, the coverage and capacity of both systems may suffer due to the so-called cross-layer interference, especially in the absence of appropriate crosslayer frequency coordination [3]. Specifically, there are two worst-case regions in which the active users may suffer from unacceptable interference and may even create a coverage hole, which detrimentally affects the macrocell users in the Cell-Edge Region (CER) and the femtocell users in the CellCentre Region (CCR) [3].

Prior research of twin-layer cellular structures characterized both the UpLink (UL) and DownLink (DL) scenarios. Some of these contributions [2]-[4] derived the Outage Probability (OP) relying on the shared spectrum access policy by considering the coverage issues. In contrast to the shared spectrum access policy, several authors [5], [6] considered assigning orthogonal spectral resources to the central marcocells and

The financial support of the RC-UK under the auspices of the India-UK Advanced Technology Centre (IU-ATC) and that of the EPSRC under the China-UK science bridge as well as that of the EU's Concerto project is gratefully acknowledged. nomadic femtocells in order to eliminate their cross-layer interference. In addition to the above-mentioned centralised approaches, the authors of [7] proposed a distributed and self-organizing femtocells management scheme conceived for Orthogonal Frequency Division Multiple Access (OFDMA)based cellular networks.

Most of the contributions in the literature stipulated the implicit assumption of Unity Frequency Reuse (UFR) aided macrocells, while there is a paucity of contributions on Fractional Frequency Reuse (FFR) aided macrocells, when hosting femtocells. Having said this, the authors of [8] studied the femtocell spectrum access problems in FFR aided macrocells, where the femtocells considered the same shared spectrum partitioning as the over-sailing marcocells in both the CCR and CER. As a result of the shared spectrum, the near-far effects still adversely affected the network performance.

Against the above background, our new contributions are:

- we propose a frequency-swapped spectrum allocation for the femtocells over-sailed by the FFR aided macrocells for the sake of overcoming the adverse near-far effects and cross-layer interference.

- we employ stochastic geometry [9], [10] for modelling the random distribution of femtocells and derive the approximate per-layer $O P$ for both the benchmarker UFR environment as well as for the proposed swapped spectrum access policy in a FFR environment.

The rest of this paper is organized as follows. In Section II, we outline our system model, while in Section III, the per-layer OP formulas are derived, followed by our results in Section IV. Finally, we offer our conclusions in Section V.

\section{System MODEL}

\section{A. Topology Model}

The topology of twin-layer cellular networks is illustrated in Fig. 1, where the over-sailing macrocells are overlaid on top of the femtocells. We model the macrocells on a regular 2$\mathrm{D}$ hexagonal lattice with a radius of $R_{c}$ and coverage area of $|\mathcal{C}|=\frac{3 \sqrt{3}}{2} R_{c}^{2}$, where the central target Macrocell Base Station (MBS) $B_{0}$ is surrounded by one ring of interfering immediately adjacent MBSs. The Femtocell Base Stations (FBS) are randomly distributed according to a homogeneous Spatial Possion Point Process (SPPP) according to an areadensity of $\lambda$ and the coverage area of each FBS is assumed to be a circle having a radius of $R_{f}$. We denote the MBS set by $\Phi$, while the FBS set by $\Psi$ and assume symmetry across the network, where every macrocell and femtocell has the same configuration. Furthermore, we assume that the femtocells 


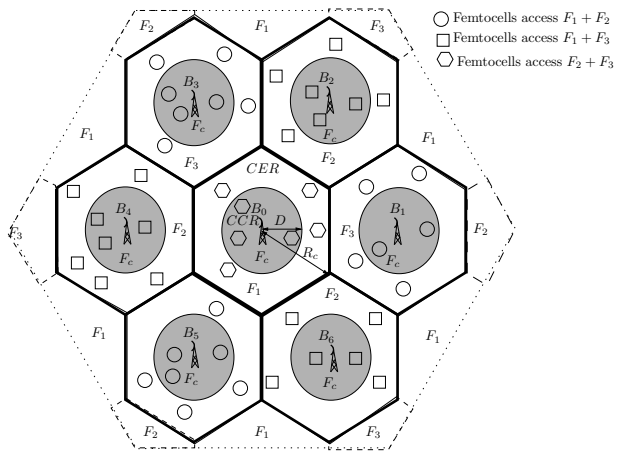

Fig. 1. The topology of frequency-swapped femtocells with FFR aided oversailing macrocells

operate on the basis of the 'Closed Access' regime of [1], where only a fixed set of subscribing home users are allowed to connect to the femtocells. Hence, the average number of femtocells per macrocell is given by $N_{f}=\lambda|\mathcal{C}|$.

\section{B. Spectrum Access Model}

In this paper, we assume a typical FFR regime for the macrocells and a swapped spectrum access for femtocells. As shown in Fig. 1, the coverage area of FFR-aided macrocells is divided into two sub-regions, namely the CCR and CER. The total available bandwidth $F$ is partitioned into four orthogonal frequency bands, $F_{c}, F_{1}, F_{2}$ and $F_{3}$, obeying $F=F_{c}+$ $F_{1}+F_{2}+F_{3}$, where $F_{c}$ represents the bandwidth available for the CCR, while $F_{i}, i \in[1,2,3]$ represents the bandwidth available for the CER of one of the three adjacent cells. In our design, the femtocells rely on the proposed swappedspectrum access, where the femtocells use frequency bands, which are orthogonal to those of the outdoor macrocell users. Specifically, the femtocells reuse the frequency band that was not assigned in its hosting macrocell. For the central cell as an example, the per-layer spectrum access under our proposed scheme is shown in last two rows of Table I.

\section{Channel Model}

In this paper, we assume that the DL channel is subject to small-scale Rayleigh fading with a unity average power, to as well as wall-penetration loss, to thermal noise and to propagation loss, where we adopt the COST231 model to characterize the propagation loss for the following scenarios:

1) MBS to cellular users: The DL pathloss between the MBS $B_{0}$ and the served cellular users is modelled as: $L_{c, d B}=$ $A_{c, d B}+10 \alpha \log _{10}(d)$, where $\alpha$ and $d$ denote the pathloss exponents (outdoor) and the distance between the macrocell users as well as the serving MBS, respectively. Furthermore, $A_{c, d B}=33.26 \cdot \log _{10}\left(f_{c}\right)-79.86$ represents the pathloss in $\mathrm{dB}$ with $f_{c}$ being the carrier frequency in $\mathrm{MHz}$.

2) FBS to home users: The DL pathloss between the FBS and the subscribing home users is modelled as: $L_{f, d B}=$ $A_{f, d B}+10 \beta \log _{10}(r)$, where $A_{c, d B}=37, \beta$ and $r$ denote the fixed pathloss in $\mathrm{dB}$, the pathloss exponents (indoor) and the distance from the users to the serving FBS, respectively.
3) MBS to home users: The DL pathloss between the MBS $B_{0}$ and the subscribing femtocell home user is modelled as: $L_{f, c, d B}=A_{f, c, d B}+10 \alpha \log _{10}\left(d_{c}\right)+W_{d B}$, where we have $A_{f, c, d B}=33.26 \cdot \log _{10}\left(f_{c}\right)-79.86$, while $\alpha, d_{c}$ and $W_{d B}$ denote the fixed pathloss in $\mathrm{dB}$, the pathloss exponents (outdoor, outdoor-to-indoor and indoor-to-outdoor), the distance between the femtocell user as well as the MBS and the wallpenetration loss in $\mathrm{dB}$, respectively.

4) FBS to cellular users: The DL pathloss between the FBS and the outdoor macrocell users is modelled as: $L_{c, f, d B}=$ $A_{c, f, d B}+10 \alpha \log _{10}\left(r_{c}\right)+W_{d B}$, where $A_{c, f, d B}=33.26$. $\log _{10}\left(f_{c}\right)-79.86$, while $\alpha, r_{c}$ and $W_{d B}$ denote the fixed pathloss in $\mathrm{dB}$, the pathloss exponents (outdoor, outdoorto-indoor and indoor-to-outdoor), the distance between the outdoor macrocell user as well as the nearest FBS and the wall-penetration loss in $\mathrm{dB}$, respectively.

5) FBS to neighbouring femtocell home users: The DL pathloss between the FBS and the neighbouring femtocell user is modelled as: $L_{f, f, d B}=A_{f, f, d B}+10 \alpha \log _{10}\left(r_{f}\right)+2 W_{d B}$, where $A_{f, f, d B}=33.26 \cdot \log _{10}\left(f_{c}\right)-79.86, \alpha, r_{f}$ and $W_{d B}$ denote the fixed pathloss in $\mathrm{dB}$, the pathloss exponents (outdoor, outdoor-to-indoor and indoor-to-outdoor), the distance between the femtocell user as well as the neighbouring FBS and the wall-penetration loss in $\mathrm{dB}$, respectively.

In this paper, we only use $A_{c}$ to represent the outdoor fixed pathloss. We assume the tagged BS and tagged user experience only Rayleigh fading and the power of the small-scale fading follows an exponential distribution with mean 1. More detailed system parameters are summarized in Table I.

\section{Per-Layer Outage Probability}

In this section, we derive the OP for both the outdoor macrocell users and the indoor femtocell users under both the traditional shared spectrum access in a UFR environment (hereafter benchmarker scenario) and in our proposed solution in a FFR-aided environment (hereafter the proposed scenario). Let us assume that the DL transmission power of each MBS and FBS is allocated by $P_{c}$ and $P_{f}$, respectively. We consider an outdoor reference cellular user at a distance of $d$ from the target central MBS $B_{0}$. We also consider a reference FBS $A_{0}$ at a distance of $d_{f}$ from the target central MBS $B_{0}$ as well as an indoor home user at a distance of $r$ and of $d_{c}$ from the FBS $A_{0}$ and the MBS $B_{0}$, respectively. Since the radius $R_{f}$ of the femtocell is significantly smaller than that of the macrocell $R_{c}$, we assume that $d_{c} \approx d_{f}$.

\section{A. Outdoor Macrocell Users' Outage Probability}

1) Benchmarker Scenario: The outdoor macrocell user may suffer from the interference imposed both by the nearby FBSs and by the other neighbouring macrocells, as well as the noise. The Signal-to-Interference-plus-Noise-Ratio (SINR) $\eta$ of the reference outdoor macrocell user may be written as:

$$
\eta(d)=\frac{\frac{P_{c}}{A_{c}} d^{-\alpha} h_{c}}{\sum_{i \in \Phi \backslash B_{0}} \frac{P_{c}}{A_{c}} d_{i}^{-\alpha} h_{i}+\sum_{j \in \Psi} \frac{P_{f}}{A_{c, f} W} r_{c, j}^{-\alpha} g_{j}+F N_{0}},
$$

where $A_{c}$ is the fixed propagation loss, $d_{i}$ and $r_{c, j}$ are the distance from the reference macrocell user to the $i$ th interfering 
MBS and the $j$ th interfering FBS, respectively, while $W$ is the wall-penetration loss in $\mathrm{dB}$. Furthermore, $h$ and $g$ denote the exponentially distributed channel gain of unity mean from the MBS and FBS, respectively. An outage occurs, when the instantaneous received SINR of a transmission falls below the target threshold $T$. With the aid of Theorem 1, we can derive the OP of the outdoor macrocell user for the benchmarker.

Theorem 1: Given the distance $d$ from the MBS $B_{0}$ and the target SINR of $T$, the OP of the reference outdoor macrocell user for the benchmarker scenario in the UFR environment may be written as:

$$
\begin{aligned}
& \mathbb{P}_{\text {out }, U F R}^{c}(T, d)=1-\exp \left[-\pi d^{2} \lambda \rho(T, K, \alpha)\right] \\
& \quad \times \exp \left[-T d^{\alpha} \frac{A_{c} F N_{0}}{P_{c}}-\frac{2 d^{\alpha} T}{(\alpha-2) R c^{2}(2 R c-d)^{2}}\right],
\end{aligned}
$$

where $K=\frac{P_{f}}{P_{c} W}$ and $\rho(T, K, \alpha)=(T K)^{\frac{2}{\alpha}} \int_{0}^{\infty} \frac{1}{1+u^{\frac{2}{\alpha}}} d u$.

Proof: The SINR of the outdoor macrocell user in Eq (1) is rewritten as $\eta(d)=\frac{h_{c}}{d^{\alpha} A_{c} F N_{0} / P_{c}+d^{\alpha}\left(I_{1}+K I_{2}\right)}$, where we have $K=\frac{P_{f}}{P_{c} W}, I_{1}=\sum_{i \in \Phi \backslash B_{0}} d_{i}^{-\alpha} h_{i}$ and $I_{2}=\sum_{j \in \Psi} r_{c, j}^{-\alpha} g_{j}$. Then the Complementary Cumulative Distribution Function (CCDF) of the outdoor macrocell user's SINR at a distance of $d$ from the MBS $B_{0}$ may be written as:

$$
\begin{aligned}
& \mathbb{P}[\eta(d) \geq T]=\mathbb{P}\left[h_{c} \geq T d^{\alpha} \frac{A_{c} F N_{0}}{P_{c}}+T d^{\alpha}\left(I_{1}+K I_{2}\right)\right] \\
& =\exp \left(-T d^{\alpha} \frac{A_{c} F N_{0}}{P_{c}}\right) \mathcal{L}_{I_{1}}\left(T d^{\alpha}\right) \mathcal{L}_{I_{2}}\left(T d^{\alpha} K\right)
\end{aligned}
$$

We let $\mathcal{L}_{I_{1}}(\cdot)$ denote the Laplace transform of the variable $I_{1}$. Here we use the Fluid model of [11] to convert the discrete MBS interference into a continuum of transmitters, hence, $\mathcal{L}_{I_{1}}$ may be formulated as:

$$
\begin{aligned}
\mathcal{L}_{I_{1}}(s) & \approx \exp \left(-s \sum_{i \in \Phi \backslash B_{0}} d_{i}^{-\alpha}\right) \\
& =\exp \left[-\frac{2 s}{\left(\alpha_{1}-2\right) R c^{2}(2 R c-d)^{2}}\right],
\end{aligned}
$$

where we approximated the instantaneous MBS interference by the long-term averaged power of MBS interference, which led to the approximation (5). Eq. (6) follows from the Fluid model presented in [11]. Futhermore, let us denote $\mathcal{L}_{I_{2}}(\cdot)$ by the Laplace transform of the Poissionian shot-noise process $I_{2}=\sum_{j \in \Psi} r_{c, j}^{-\alpha} g_{j}$, which is given as:

$$
\begin{aligned}
\mathcal{L}_{I_{2}}(s) & =\mathbb{E}_{I_{2}}\left[\exp \left(-s \sum_{j \in \Psi} r_{c, j}^{-\alpha} g_{j}\right)\right] \\
& =\mathbb{E}_{\Psi, g_{j}}\left\{\prod_{j \in \Psi} \mathbb{E}_{g_{i}}\left[\exp \left(-s g_{i} r_{c, j}^{-\alpha}\right)\right]\right\} \\
& =\mathbb{E}_{\Psi}\left(\prod_{j \in \Psi} \frac{1}{1+s r_{c, j}^{-\alpha}}\right) \\
& \stackrel{a}{=} \exp \left[-2 \pi \lambda \int_{0}^{\infty}\left(1-\frac{1}{1+s x^{-\alpha}}\right) x d x\right],
\end{aligned}
$$

where we have $s=T d^{\alpha} K$. The equality (a) in Eq (10) follows from the Probability Generating Functional (PGFL) of the SPPP. Specifically, when having a pathloss exponent of $\alpha=4$, then Eq. (10) may be rewritten as: $\mathcal{L}_{I_{2}}\left(T d^{\alpha} K\right)=$ $\exp \left[-\frac{\pi^{2}}{2} d^{2} \lambda(T K)^{\frac{1}{2}}\right]$. Substituting Eq. (6) and Eq. (10) into Eq. (4) gives the desired result, which is written as:

$$
\mathbb{P}_{\text {out }, U F R}^{c}(T, d)=1-\mathbb{P}[\eta(d) \geq T] .
$$

2) Proposed Solution: When employing the proposed swapped-spectrum access, the outdoor macrocell user roaming in the CCR suffers from the interference imposed by the neighbouring MBSs, as well as from the noise. As a result, the SINR $\eta_{C C R}$ of the outdoor macrocell users located in the CCR may be written as:

$$
\eta_{C C R}=\frac{\frac{P_{c}}{A_{c}} d^{-\alpha} h_{c}}{\sum_{i \in \Phi \backslash B_{0}} \frac{P_{c}}{A_{c}} d_{i}^{-\alpha} h_{i}+F_{c} N_{0}} .
$$

The outdoor macrocell users roaming in the CER may only suffer from the effects of FBSs located in the other macrocells and the noise, hence we may formulate the SINR $\eta_{C E R}$ of the outdoor macrocell user in the CER as:

$$
\eta_{C E R}=\frac{\frac{P_{c}}{A_{c}} d^{-\alpha} h_{c}}{\sum_{j \in \Psi^{\prime}} \frac{P_{f}}{A_{c, f} W} r_{c, j}^{-\alpha} g_{j}+F_{1} N_{0}},
$$

where $\Psi^{\prime}$ denotes the set of femtocells located in other macrocells. Hence the corresponding OP of the outdoor macrocell users is formulated in Theorem 2 for our proposed solution

Theorem 2: Given the distance $d$ from the MBS $B_{0}$ and the target SINR threshold of $T$, the OP of the reference outdoor macrocell user for our proposed spectrum-swapping solution used in the FFR environment may be formulated as in Eq. (15).

Proof: Comparing Eq. (12) to Eq. (1), the OP of outdoor macrocell users located in CCR is simply derived by removing $\mathcal{L}_{I_{2}}$ of Eq. (2). For the CER users, the FBS interference may be dominated by the FBSs located in the nearest macrocells. We observed that the FBS interference imposed on the target macrocell users is dominated by the FBSs located in the angle range ranging from $-\frac{\pi}{3}$ to $\frac{\pi}{3}$ (assuming that the link between the MBS $B_{0}$ and the macrocell user is a horizontal line). Our simulations show that this observation is valid when $d<0.95 R_{c}$. Similarly to $I_{2}$, we have $I_{3}=\sum_{j \in \Psi \prime} r_{c, j}^{-\alpha} g_{j}$, and as a result, $\mathcal{L}_{I_{3}}$ is written as:

$$
\mathcal{L}_{I_{3}}(s)=\exp \left[-\lambda \frac{2 \pi}{3} \int_{\frac{\left(R_{c}-d\right)^{2}}{d^{2} \sqrt{T K}}}^{\infty}\left(1-\frac{1}{1+s x^{-\alpha}}\right) x d x\right]
$$

Hence, assuming $\alpha=4$, the OP of CER users is given by Eq. (15).

\section{B. Indoor Femtocell Users' Outage Probability}

For the indoor femtocell users, the interference from the neighboring MBSs and the FBSs located in neighboring macrocells will be ignored. We also assume that the noise effects are negligible when compared to those of the interference from the over-sailing MBS and nearby FBSs. Our simulation results will show the accuracy of this approximation. 


$$
\begin{gathered}
\mathbb{P}_{\text {out }, F F R}^{c}(T, d)= \begin{cases}1-\exp \left[-d^{\alpha} \frac{A_{c}}{P_{c}} F_{c} N_{0} T-\frac{2 d^{\alpha} T}{(\alpha-2) R c^{2}(2 R c-d)^{2}}\right], & \text { if } d \leq D, \\
1-\exp \left[-d^{\alpha} \frac{A_{c}}{P_{c}} F_{1} N_{0} T-\lambda d^{2} \sqrt{T K}\left(\frac{\pi^{2}}{6}-\frac{\pi}{3} \arctan \frac{\left(R_{c}-d\right)^{2}}{d^{2} \sqrt{T K}}\right)\right], & \text { if } D<d \leq R_{c}\end{cases} \\
\mathbb{P}_{\text {out }, U F R}^{f}\left(T, d_{f}\right)=1-\frac{2}{R_{f}^{2}} \int_{0}^{R_{f}} \frac{\exp \left[-\pi r^{\beta / 2} \lambda \rho(T, J, \alpha)\right]}{1+T \frac{P_{c} A_{f}}{P_{f} A_{c} W} d_{f}^{-\alpha} r^{\beta}} r d r
\end{gathered}
$$

1) Benchmarker Scenario: In this scenario, the indoor home user residing within the coverage of the FBS $A_{0}$ may suffer from the interference imposed both by the MBSs and by the nearby FBSs, as well as from the noise. As a result, the SINR $\eta$ of an indoor home user within the coverage of the reference FBS $A_{0}$ may be formulated as:

$$
\eta\left(d_{f}, r\right)=\frac{\frac{P_{f}}{A_{f}} r^{-\beta} g_{f}}{\frac{P_{c}}{A_{c}} d_{c}^{-\alpha} h_{i}+\sum_{j \in \Psi \backslash A_{0}} \frac{P_{f}}{A_{f, f} W^{2}} r_{f, j}^{-\alpha} g_{j}} .
$$

The OP of the indoor home user communicating in the benckmarker scenario is given by Theorem 3. The corresponding proof is similar to that of Theorem 1 .

Theorem 3: Given the distance $d_{f}$ from the reference FBS $A_{0}$ to the MBS $B_{0}$ and the target SINR threshold of $T$, the OP of the indoor home user communicating in the benchmarker scenario may be expressed as Eq. (16), where we have $J=$ $\frac{A_{f}}{A_{c} W^{2}}$ and the function $\rho(\cdot)$ is given in Theorem 1 .

Specifically, assuming outdoor and indoor pathloss exponent of $\alpha=4$ and $\beta=2$, respectively, the OP of the indoor home user is formulated as:

$$
\mathbb{P}_{\text {out }, U F R}^{f}\left(T, d_{f}\right)=1-\frac{2 H}{R_{f}^{2}} .
$$

where $H$ can be derived from [12], and written as:

$$
\begin{array}{r}
H=\frac{1}{z}\left\{e^{\frac{i y}{\sqrt{z}}}\left[E i\left(x y-\frac{i y}{\sqrt{z}}\right)-E i\left(\frac{i y}{\sqrt{z}}\right)\right]\right. \\
\left.+e^{\frac{-i y}{\sqrt{z}}}\left[E i\left(x y-\frac{i y}{\sqrt{z}}\right)-E i\left(\frac{i y}{\sqrt{z}}\right)\right]\right\},
\end{array}
$$

where $\mathrm{Ei}$ is the exponential integral function, with $x=R_{f}$, $y=-\frac{\pi^{2}}{2} \lambda \sqrt{T J}$ and $z=T \frac{P_{c} A_{f}}{P_{f} A_{c} W} d_{f}^{-4}$.

2) Proposed Solution: Under our proposed solution, the target femtocell $A_{0}$ 's indoor home user endures the interference from the nearby FBSs, denoted as $\Psi \backslash A_{0}$. As a result, the SINR $\eta_{C C R}$ of the indoor home user may be expressed as:

$$
\eta\left(d_{f}, r\right)=\frac{\frac{P_{f}}{A_{f}} r^{-\beta} g_{f}}{\sum_{j \in \Psi \backslash A_{0}} \frac{P_{f}}{A_{f, f} W^{2}} r_{f, j}^{-\alpha} g_{j}^{2}} .
$$

Theorem 4 characterizes the OP of the indoor home user of the proposed spectrum-swapping solution in the FFR environment.

Theorem 4: Given the distance $d_{f}$ from the reference FBS $A_{0}$ to the MBS $B_{0}$ and the target SINR threshold of $T$, the $\mathrm{OP}$ of the indoor home user communicating by employing the spectrum-swapping in the FFR environment is given by:

$$
\mathbb{P}_{\text {out }, F F R}^{f}\left(T, d_{f}\right)=1-\frac{2}{R_{f}^{2} t} A^{-\frac{2}{t}} \gamma\left(\frac{2}{t}, A R_{f}^{t}\right)
$$

where $t=\frac{\beta}{2}, A=\frac{\lambda \pi^{2}}{2}\left(\frac{T A_{f}}{A_{c} W^{2}}\right)^{\frac{1}{2}}$. Furthermore, $\gamma(s, x)$ is the incomplete gamma function, which is written as:

$$
\gamma(s, x)=\int_{0}^{x} t^{s-1} e^{-t} d t .
$$

Proof: Similarly to the proof of Theorem 1, the SINR of the indoor home user in Eq. (19) may be formulated as:

$$
\eta\left(d_{f}, r\right)=\frac{g_{f}}{r^{\beta} J I_{2}},
$$

Then the CCDF of the indoor home user's SINR may be expressed as:

$$
\mathbb{P}\left[\eta\left(d_{f}, r\right) \geq T\right]=\mathcal{L}_{I_{2}}\left(\operatorname{Tr}^{\beta} J\right),
$$

where $\mathcal{L}_{I_{2}}(\cdot)$ is given by Eq. (10).

Since the indoor home users tend to be uniformly distributed in the coverage region of their FBS, the OP of an indoor home user benefiting from our proposed solution may be written as:

$$
\begin{aligned}
\mathbb{P}_{\text {out }, F F R}^{f}\left(T, d_{f}\right) & =\mathbb{E}_{r}\left[\eta\left(d_{f}, r\right)<T \mid 0 \leq r \leq R_{f}\right] \\
& =1-\frac{2}{R_{f}^{2}} \int_{0}^{R_{f}} r \mathcal{L}_{I_{3}}\left(T r^{\beta} J\right) d r \\
& =1-\frac{2}{R_{f}^{2} t} A^{\left(-\frac{2}{t}\right)} \gamma\left(\frac{2}{t}, A R_{f}^{t}\right) .
\end{aligned}
$$

\section{NUMERICAL RESUlts}

This section presents our numerical results generated for investigating the OP of both the benchmarker and of the proposed spectrum-swapping scenario. The system parameters are summarized in Table. I. The femtocells are generated following the SPPP, which are randomly distributed within the over-sailing macrocells. We assumed that the bandwidth available for the CCR is $F_{c}=F / 2$, and that for the CER is $F_{i}=F / 6, i=[1,2,3]$.

Fig. 2 illustrates the OP for an outdoor macrocell user as a function of the distance from the user to the central MBS, when we set the averaged number of FBSs per cell to be $N_{f}=10$ and $N_{f}=100$, respectively. Observe in Fig.2 that as expected, the OP increases, as the user is moving from the CCR to the CER. More explicitly, the OP becomes higher than $0.5\left(N_{f}=100\right)$ if we have $d \geq 0.55 R_{c}$ for the benchmarker in a UFR environment. As a result, the outdoor users in the CER may consistently suffer from transmission outages, owing to the significant interference impinging from both the nearby FBSs and from the neighbouring MBSs. By contrast, the OP of an outdoor macrocell user is significantly reduced for our proposed spectrum-swapping scenario in the 
TABLE I

NOTATIONS AND SYSTEM PARAMETERS

\begin{tabular}{|l|l|r|}
\hline Symbol & Description & Value \\
\hline$\Phi$ & MBS set & N/A \\
\hline$\Psi$ & FBS set & N/A \\
\hline$f_{c}$ & Carrier frequency & $2 \mathrm{GHz}$ \\
\hline$N_{0}$ & Thermal noise density & $-174 \mathrm{dBm} / \mathrm{Hz}$ \\
\hline$R_{c}$ & Radius of the Macrocell & $1000 \mathrm{~m}$ \\
\hline$D$ & Radius of the CCR & $\frac{2}{3} R_{c}$ \\
\hline$F$ & Total available bandwidth & $10 \mathrm{MHz}$ \\
\hline$R_{f}$ & Radius of the Femtocell & $20 \mathrm{~m}$ \\
\hline$N_{f}$ & Number of FBSs per cell & 10,100 \\
\hline$A_{c, d B}$ & Fixed outdoor pathloss & $33.26 \log _{10}\left(f_{c}\right)-79.86 \mathrm{~dB}$ \\
\hline$A_{f, d B}$ & Fixed indoor pathloss & $37 \mathrm{~dB}$ \\
\hline$\alpha$ & Outdoor pathloss exponent & 4 \\
\hline$\beta$ & Indoor pathloss exponent & $5 \mathrm{~dB}$ \\
\hline$W_{d} B$ & Wall penetration loss & $46 \mathrm{dBm}$ \\
\hline$P_{c, d B}$ & Transmit power & $13 \mathrm{dBm}$ \\
\hline$P_{f, d B}$ & Transmit power & $0 \mathrm{~dB}, 30 \mathrm{~dB}$ \\
\hline$T_{d B}$ & Target SINR threshold & Femtocell \\
\hline \hline & Macrocell & $F_{2} \& F_{3}$ \\
\hline $\mathrm{CCR}$ & $F_{c}$ & $F_{2} \& F_{3}$ \\
\hline $\mathrm{CER}$ & $F_{1}$ &
\end{tabular}

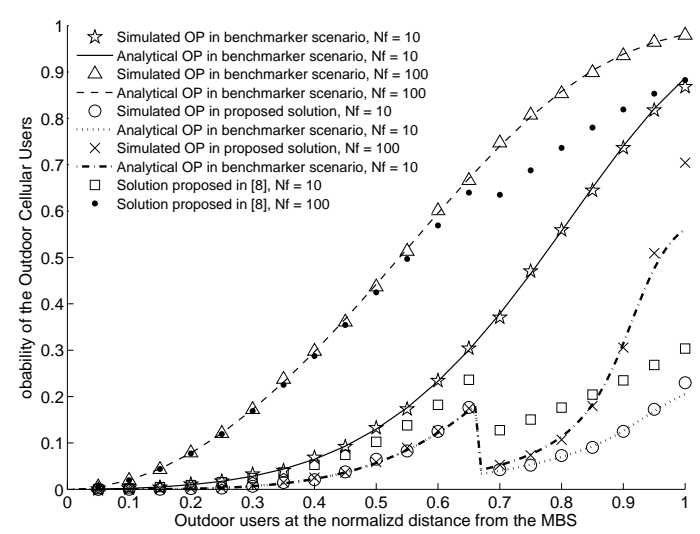

Fig. 2. The outage probability of outdoor macrocell users according to the their normalized distance from the MBS, for the target threshold of OdB and using the parameters of Table I.

FFR environment. Our proposed scheme also outperforms the solution advocated in [8] in both the CCR and CER. Additionally, we also verified the formulas derived in our paper with the aid of simulation results. We demonstrated that our theoretical results are accurate in most distance regions $\left(d<0.95 R_{c}\right)$ and hence they are capable of quantitatively characterizing the $\mathrm{OP}$ as a function of the distance from the central MBS in both cases.

Fig. 3 shows the OP of an indoor home user as a function of the distance from the FBS to the central MBS, when we set the averaged number of FBSs per cell to be $N_{f}=10$ and $N_{f}=100$, respectively. For the benckmarker, the indoor users may suffer from a high OP, when their serving FBS is near to the MBS. However, when the FBS is sufficiently far away from the MBS, the OP is rapidly reduced. Finally, the OP of the indoor users supported by our spectrum-swapping regime remains low, because the interference emanating form the neighbouring FBSs is significantly reduced owing to the attenuation of walls. Compared to the benchmarker scenario,

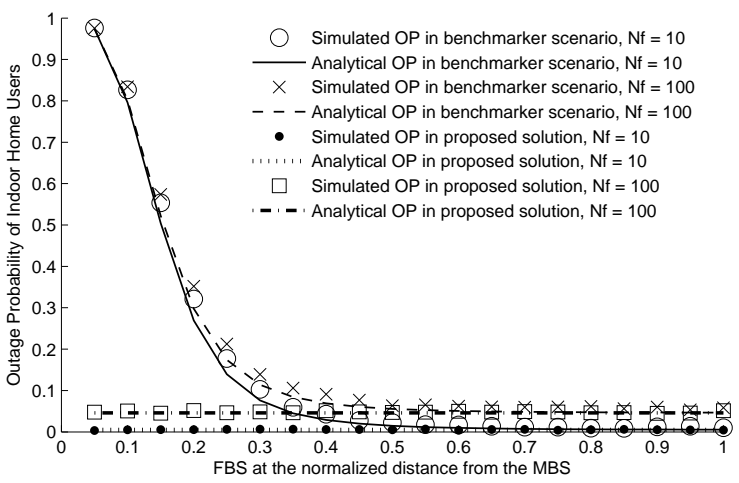

Fig. 3. The outage probability of indoor home users according to the distance from their serving FBS to the MBS, for the target threshold $30 \mathrm{~dB}$ and using the parameters of Table I.

the solution proposed in [8] would not reduce the OP for indoor users, hence we only present the OP of the benchmarker scenario. We also observe that our analytical OP formulae accurately predict the OP, as confirmed by our simulations.

\section{CONCLUSIONS}

In this treatise, we investigated the OP of twin-layer cellular networks, where the MBSs employing FFR host the FBSs and we derived closed-form expressions for the OP of outdoor macrocell users and indoor home users, respectively. The simulation results show that the proposed swapped spectrum access policy is capable of overcoming the typical near-far problem and our analytical results accurately predict the OP.

\section{REFERENCES}

[1] V. Chandrasekhar and J. G. Andrews, "Femtocell Networks: A Survey," IEEE Communications Magazine, vol. 46, pp. 59-67, Sep. 2008.

[2] V. Chandrasekhar and J. G. Andrews, "Uplink Capacity and Interference Avoidance for Two-Tier Femtocell Networks," IEEE Transactions on Wireless Communications, vol. 8, pp. 3498-3509, July 2009.

[3] V. Chandrasekhar, M. Kountouris, and J. G. Andrews, "Coverage in Multi-Antenna Two-Tier Networks," IEEE Transactions on Wireless Communications, vol. 8, pp. 5314-5327, Oct. 2009.

[4] G. Jeney, "Practical Limits of Femtocells in a Realistic Environment," in Proc. IEEE VTC., pp. 1-5, May 2011.

[5] V. Chandrasekhar and J. G. Andrews, "Spectrum Allocation in Tiered Cellular Networks," IEEE Transactions on Information Theory, vol. 57, pp. 3059-3068, Oct. 2009.

[6] K. Yeung and S. Nanda, "Channel Management in Microcell/Macrocell Cellular Radio Systems," IEEE Transactions on Vehicular Technology, vol. 45, pp. 601-612, Nov. 1996.

[7] J. Yun and K. G. Shin, "Adaptive Interference Management of OFDMA Femtocells for Co-Channel Deployment," IEEE Journal on Selected Areas in Communications, vol. 29, pp. 1225-1241, June 2011.

[8] Z. Zhao, F. Zheng, A. Wilzeck, and T. Kaiser, "Femtocell Spectrum Access Underlaid in Fractional Frequency Reused Marcocell," in Proc, IEEE ICC. Workshop, pp. 1-6, June 2011.

[9] M. Haenggi, J. G. Andrews, F. Baccelli, O. Dousse, and M. Franceschetti, "Stochastic Geometry and Random Graphs for the Analysis and Design of Wireless Networks," IEEE Journal on Selected Areas in Communications, vol. 27, pp. 1029-1046, Sep. 2009.

[10] F. Baccelli, B. Blaszczyszyn, and P. Muhlethaler, "Stochastic Analysis of Spatial and Opportunistic Aloha," IEEE Journal on Selected Areas in Communications, vol. 27, pp. 1105-1119, Sep. 2009.

[11] J. M. Kelif, M. Coupechoux, and P. Godlewski, "A Fluid Model for Performance Analysis in Cellular Networks," EURASIP Journal on Wireless Communications and Networking, vol. 2010, Jun. 2010.

[12] I. S. Gradshteyn and I. M. Ryzhik, Table of Integrals, Series, and Products. 